\title{
Adaptive and Cognitive Communication Architecture for Next- generation PPDR Systems
}

\author{
Ozgur Ergul ${ }^{1}$, Ghalib A. Shah ${ }^{2}$, Berk Canberk ${ }^{3}$, Ozgur B. Akan ${ }^{1}$
}

\author{
${ }^{1}$ Next-generation and Wireless \\ Communications Laboratory, \\ Koc University, \\ Istanbul Turkey 34450
}

\author{
${ }^{2}$ Al-Khawarizmi Institute of Computer \\ Science, \\ University of Engineering and \\ Technology, \\ Lahore Pakistan 54000
}

\author{
${ }^{3}$ Broadband Communication Research \\ Group \\ Istanbul Technical University \\ Istanbul Turkey 34469
}

In the light of the recent natural catastrophes and terrorist activities it has become evident that new architectural approaches are needed for the next generation Public Protection and Disaster Relief (PPDR) networks. These architectures should be adaptable to the conditions at the event site, resilient enough to operate under adverse conditions of the emergency. Furthermore, they should enable timely gathering of crucial event data and its delivery to the responder units at the site as well as the command and control centre that are off-site. In this paper, we first examine the stateof-the-art for areas related to communication in PPDR systems, and discuss the open research issues for each topic. Then, we propose a novel architecture that meets the aforementioned requirements which relies on a novel device called Intelligent Cognitive Gateway (ICG). ICG enables flexible use of the spectrum and facilitates data gathering from all lower tier devices and relays this data to the relevant units through the higher tier public or commercial backhaul networks. Finally, we provide some results that justify the need for these devices in emergency scenarios.

\section{Introduction}

When responding to emergency situations, the quality and sustainability of the communication have great impact on the performance of the first responders. Recent natural catastrophes (e.g. Hurricane Katrina) and terrorist activities (e.g. London metro bombings) revealed that even the most recent TETRA/TERAPOL systems have inadequacies.

The current PPDR networks provide feature-rich voice centric services but they offer a very limited range of data services for imaging, video, and data files. Recently, Tetra Release 2, Tetra Enhanced Data Services (TEDS) was introduced. Though some efforts have been devoted to upgrade the existing standards with wideband data capabilities, the development towards an enhanced mobile broadband solution for public safety lags far behind the commercial mobile broadband wireless networks [1]. Moreover, today's immediate missing requirement is interoperability, not only between different services, but also within the same service if different systems are in operation between regions [2]. Furthermore, TETRA/TETRAPOL networks have a cell-based central structure and failure in a base station leads to large coverage loss and possibly network partitioning.
To address these inadequacies there has been an increasing amount of work in the literature on network resilience and PPDR networks. Our aim in this paper is two-fold. We first, classify and examine the previous work in the literature, discussing open research venues for each sub topic. Then we focus on research related to data retrieval during and after an emergency and introduce a novel, multi-tier, cognitive communication architecture for adaptive and sustainable wireless communication required in PPDR operations.

So far, the existing works on communication in PPDR systems have only partially addressed the problem of providing uninterrupted/sustainable communication. Some propose a dedicated network for public safety operation such as TETRA/TETRAPOL, while others rely on the existing commercial networks [3]. Although some cognitive radio (CR) architectures [4] are also proposed to countermeasure the failure of the existing networks, all these efforts are made in a non-collaborative manner and there is a need to intelligently integrate them and address different issues in a focused way.

\section{Previous Work}

One of the greatest problems in current networks is the lack of resilience. The research on network resilience can broadly be divided into two as research aiming pre and post emergency.

\section{Research on Pre-disaster Improvements}

Most of the research in the literature on network resilience and specifically on PPDR networks may be included among improvements that can be made before the emergency. These mainly include

a. resilience analysis,

b. resilient network design

i. resilient backhaul network design

ii. resilient access network design

c. developing resilient network layers, or modifying existing network layers to introduce resilience.

\section{Resilience Analysis}

Resilience analysis research includes finding meaningful metrics to analyze networks in terms of failure types, and developing algorithms that can generate resilient network topologies. Moreover, conventional performance metrics are not suitable for mission-critical networks [5]. With these metrics, high probability events dominate their effect on the performance measures. However, in mission-critical networks, 
certain events with rare occurrences may have dramatic importance. For example, if link sustainability is taken as an important metric, resulting design may prioritize finding channels that retain desirable conditions. However, in case of a terrorist attack, an adaptive jammer that looks for good channels to jam may render these solutions highly ineffective.

European Network and Information Security Agency (ENISA) issued a report on resilience metrics [6]. The report does not propose new metrics but rather, lays out an overview of previous work on the subject. It is pointed out that measuring the effectiveness of current resilience policies is challenging, and the discipline is still in its early stages.

No consensus is developed on the identification of metrics and standards for measuring resilience. United Nations Development Programme (UNDP) has recently conducted a survey over the resilience measures taken across the world [7]. The report indicates that resilience has various elements (e.g. well-being, vulnerability, etc.), levels (e.g. inputs, impacts ,etc.), dimensions (e.g. technical, economical, etc.). Furthermore, measurements may be tailored to context (e.g. Country Disaster Resilience Index for coastal communities). The conclusion of the report is that the resilience metrics and measurements should cover as many of these elements, dimensions, etc. as possible.

\section{Resilient Network Design}

Resilient network design research has mostly been on backhaul networks. Standards such as IEEE 802.17 on Resilient Packet Ring (RPR) address resilience directly. Amendments to existing standards, such as IEEE 802.3ah (Ethernet Passive Optical Networks) and recommendations, such as ITU-T G.984 (gigabit passive optical networks) help in developing backhaul networks that remain operational in case of power shortages. These approaches take advantage of ability to function without electrically powered switching components.

Research on this front mostly focuses on ring or mesh topologies that introduce redundancy to obtain fault tolerance [8]. New approaches that use recently emerging network types are needed. For example, vehicle to roadside (V2R) communication is a recent hot topic. In case of backhaul network failures, these networks may be utilized. However, they are generally designed in a linear fashion and lack redundancy, thus, fault tolerance. Also, handover algorithms for V2R are straightforward due to the simple structure of the network structure. Introducing resilience to such recently developing networks either in form of network design (e.g. mesh V2R networks) or algorithm design (e.g. resilient handover algorithms) to exploit them in case of an emergency scenario is an open research area.

Software Defined Networking (SDN) is a new paradigm that simplifies network management by decoupling the decision making system on traffic forwarding (control plane) from the actual underlying system that does the forwarding (data plane). Due to this abstraction, SDN provides a very convenient means for traffic management in case of a disaster [9]. There is minimal amount of work in the literature on using
SDN to design disaster resilient backhaul networks. Algorithms that can rapidly adapt to new conditions in case of component failures and configuration changes, which may occur frequently after a disaster, are needed. Analysis and efficient methods that consider mapping of virtual resources to physical components are also among the open research issues.

When a disaster strikes, wired backhaul networks may fail due to wire cuts. Wireless backhaul networks provide a promising alternative. Generally, directional antennas are used in wireless backhaul networks to meet the high performance required. Since the number of directional antennas per unit is limited, node degree is limited. However, to increase resilience and introduce fault tolerance, high node degree is desired. New research efforts that address the trade-off between node degree, network performance and cost are needed. Another open issue is designing resilient wireless backhaul networks based on the results of these trade-off analyses.

Compared to the research on resilience of backhaul networks, the effort on designing resilient access networks is limited. Access networks may provide invaluable data for first responder units after the disaster. Consider an example case where an operational wireless access point (AP) in a collapsed building can communicate with a wearable health monitoring device in the wireless personal area network (WPAN) of a patient. By accessing the AP, first responder units may gather vital data for their rescue operations.

There are many open research venues on this topic. To name a few, methods that make use of Power Line Communications (PLC) are needed. Recently, PLC is being considered as a part of home digital networks [10]. With proper design, a PLC network with components that have backup batteries, may still be partly operational after a disaster, even in case of occasional power line cuts. Another important alternative is designing resilient femto/picocell Home Area Networks (HAN). Resilient network designs that make use of these new and emerging access networks are needed.

\section{Resilient Network Layers}

Algorithms that are developed for various network levels, such as routing algorithms, congestion control algorithms, and network coding algorithms must be reconsidered to increase traffic flow resilience in case of component failures. Most of the research on this topic is on developing countermeasures for malicious attacks [11]. However, outages due to disaster cases are different. There is limited amount of work in the literature such as [12], where a cognitive routing protocol that takes quality of service (QoS) into account is proposed. Opportunistic spectrum access (OSA) provided by CR may prove to be of great value for establishing communications in case of a disaster. Research on this front is very limited. Routing algorithms that consider spectrum availability, sensing algorithms that can search, identify and communicate with operational access network devices, algorithms that consider directional transmission with spectrum availability are some of the open research areas on this topic. 


\section{Research on Post-disaster Improvements}

Research on work that must be performed after the disaster mainly consist of means of data retrieval form the emergency area and forwarding this data to first responder units and their command and control centres. These can be broadly categorized as

- through-the-wall inspection

- operational device identification

- resilient data fusion and routing

\section{Through-the-wall Detection}

Firefighters and first responders use through-the-wall detection technologies to locate people in collapsed or burning buildings. The most dominant research area is ultra wide band (UWB) [13]. However, since UWB uses a very large bandwidth, its impact on wireless communication in the event area must be analysed. It was reported that even though interference from a single UWB device has negligible effect on narrowband (NB) devices, if the NB receiver is closer to the UWB transmitter than to the NB transmitter, this may cause very low Signal to Interference Ratio (SIR) and cause performance degradation in the NB link [14].

\section{Operational Device Identification}

Another important issue is to identify operational devices inside the event area. These devices can provide invaluable information to first responder units. They may be under collapsed concrete or in distant locations that first responders cannot move into due to the nature of the disaster (e.g. radiation, fire, etc.). To increase the chance of identifying and communicating with operational access network devices intelligent means of using directional antennas and fast sensing algorithms that can scan the spectrum bands of these devices are essential. There has been some work on direction of arrival
(DoA) estimation to increase the performance of directional antennas [15]. However, algorithms that coordinate scanning of bands in conjunction with DoA to discover operational devices are needed, considering that these operational devices may use a variety of access technologies (IEEE 802.15, GSM, 3G, etc.).

Radiation pattern of directional antennas differ with frequency. Methods that use CR capabilities with directional wireless communication can increase performance. These methods should also consider fading since fading varies with the communicating frequency. Therefore, more resilient and efficient communication may be possible with novel algorithms that take into account all of the aforementioned factors.

\section{Resilient Data Fusion and Routing}

When the operational wireless devices are found using the means mentioned above, algorithms to retrieve data efficiently and rapidly is essential. Cognitive Radio Sensor Networks are a relatively recent paradigm [16], which can be very useful in a PPDR network architecture. A Cognitive Radio Network can also be used to restore functionality to partially destroyed networks by providing connectivity through alternative bands as proposed in [17]. Such novel resilient data delivery and data aggregation algorithms, restorative routing algorithms that consider spectrum availability must be developed.

We present a summary of the open research issues in Table 1, for each research area. One of the overlooked capabilities in PPDR architecture is the capability to extract data from the event area, either from still functional devices in the area or from devices deployed by the first responder units, after the event. In the following section, we present a new adaptive and cognitive PPDR architecture that has the features and structure to cover this shortcoming.

\begin{tabular}{l|l|l|}
\hline Category & Research area & Open Issue \\
\hline $\begin{array}{l}\text { Pre-Disaster } \\
\text { Improvements }\end{array}$ & Resilience Analysis & Metrics suitable for mission critical networks \\
\cline { 2 - 3 } & Resilience analysis for mission-critical networks \\
\hline \multirow{2}{*}{$\begin{array}{l}\text { Resilient Network Design } \\
\text { Resilient network architectures for both backhaul and access networks }\end{array}$} \\
\hline
\end{tabular}

Table 1 Categorization of studies on PPDR 


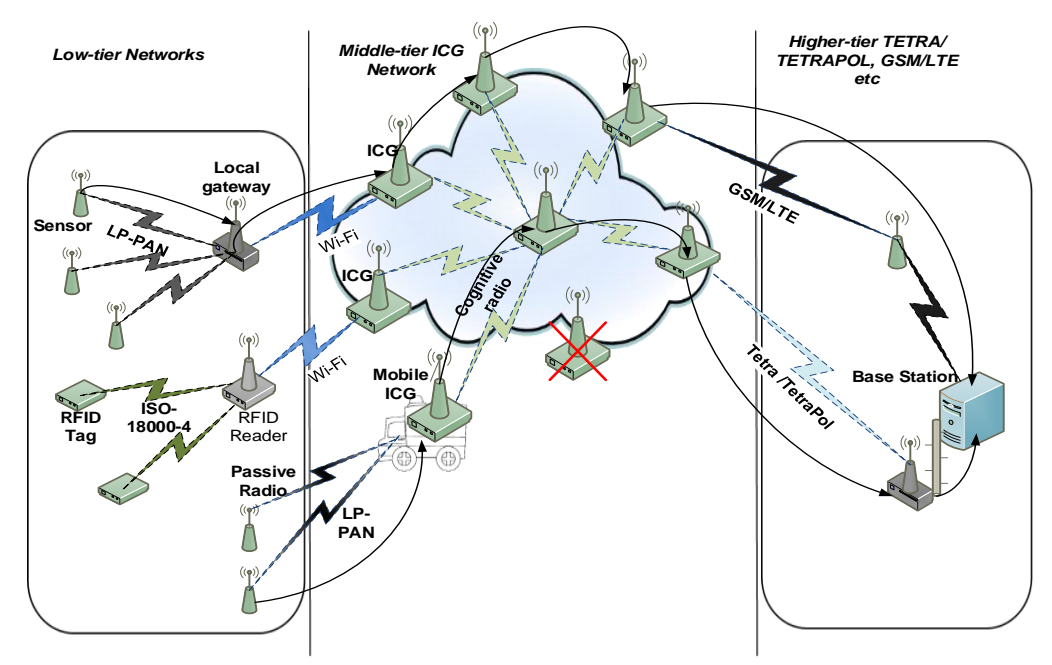

Figure 1 Proposed architecture for future PPDR Infrastructure.

\section{Adaptive and Cognitive Communication Architecture for PPDR}

Post-disaster improvements have attracted less interest from the research community, compared to pre-disaster improvements. The limited existing effort is generally focused on one aspect such as the ICT infrastructure for PPDR vehicles and satellite communication. In this section, we lay out a broader proposal for a new PPDR network architecture that aims gathering data from the event area in case of an emergency and forwards this crucial information to command units. First, we list the challenges that must be overcome by such architecture.

\section{Challenges}

Spectrum Usage: The fundamental challenge in PPDR systems is to specify the part of spectrum for exclusive use of PPDR communication. However, with new applications such as real-time video from drones, and through-the-wall imaging, the demand for bandwidth in future first responder networks will be exceptionally high and exclusive access to the spectrum may not be sufficient, since only a limited amount of bandwidth can be spared for any one service

Interoperability: In disaster scenarios, various national and international organizations perform rescue operations to cover the large incident region. This requires interoperability of the devices and equipment.

Self-organization: In order to help emergency personnel to concentrate on the tasks, incident area network should be deployed quickly, with little human maintenance. Therefore, devices must be capable of self-organizing into a network.

Reliability: Reliability is required for data to be consistently and continuously transported to the central command station or the data collection points. Also, first responders' connections amongst themselves and the command center should be reliable.
Scalability: It refers to the ability of a system to support large number of parameters without impacting the performance. These parameters include number of nodes, traffic load and mobility aspects. Limited processing and storage capacities of the radio devices are also a concern.

\section{The Network Architecture}

We propose a novel multi-tier cognitive communication architecture that can overcome these challenges. The required resilience and adaptation are provided with the concept of Intelligent Cognitive Gateways (ICGs). An ICG is a gateway with multiple interfaces to interact with both various low level devices such as sensor nodes, RFID readers, Wi-Fi routers, etc., and high level devices such as commercial and backbone network devices. ICGs provide means for OSA through their cognitive radio interface. They can be manufactured in various forms such as mobile ICGs, and ICG with the capability of passive communications. The details of the three-tiered architecture are as follows.

\section{Low Tier Application Network}

Low tier consists of wireless sensor network (WSN) nodes composed of any kind of sensing devices, remote health monitoring, telemetry and voice phones. The low tier elements are usually low power devices with short range communication capabilities.

The potential applications commonly realized in PPDR system deploy a variety of lower-tier devices used for day-today operations, as well as on-scene mobile devices. For example, a remote healthcare monitoring application can deploy ECG, asthma (Nanotube Asthma Sensor), swine flu, and diabetes sensors (Glucose sensor etc.) at low tier. Several of these low tier elements are organized in one unit and can form network nucleus interfacing to middle tier by one gateway/port only. 


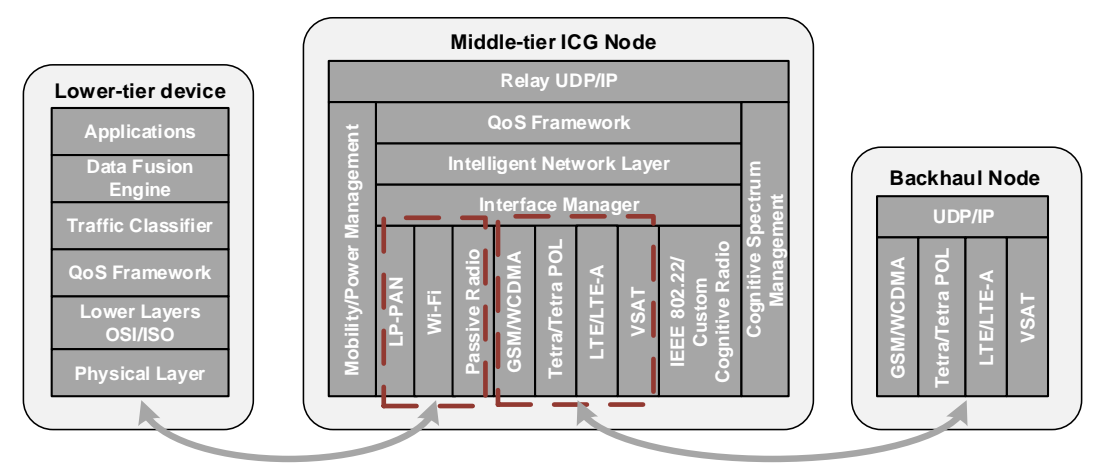

Figure 2 Communication protocol stack of the architecture.

An example of the network nucleus could be the RFID reader fetching information from large number of tags. Thus, low tier network is analogous to the WSN that builds ad hoc auto configuring architecture based on short-range transmission network. Data collected by these devices are sent to a nearby ICG directly or via multi-hop routing through neighboring nodes. Therefore, ICG integrates the most widely used low power wireless interfaces such as IEEE 802.15.4. Additionally, battery-less devices may also be used. These devices do not communicate actively. They have a passive radio interface which can modulate reflected waves. This enables the capability for mobile ICGs to move into the event area and fetch information from these passive sensing devices by sending radio waves to them.

\section{Middle-Tier PPDR Infrastructure}

ICGs form the middle-tier of the architecture and enhance the existing PPDR infrastructure. They provide close-in connectivity to lower-tier devices in order to access backhaul network. These ICG nodes are not only deployed for day-today routine operations of the evolving PPDR applications, but also allow self-configured ad hoc extension to the infrastructure in incident areas. ICGs are equipped with intelligent cognitive radio capability that can tune into any of the communication bands of the lower tier devices, recognize and authenticate nodes and provide communication interface to/from application networks. Each ICG forms a cluster with its lower tier surrounding devices.

Established standards for CR, such as IEEE 802.22 and IEEE 802.11af have certain problems when used in a first responder network. 802.22 is a centralized approach that relies on a base station. Basing the whole architecture on a BS that may not be operational is a problem. On the other hand, 802.11 af requires connection to a geolocation database (GDB) to query available bands. Such connection may not be available in an event area. However, first responders may keep copies of these databases and bring them along to be used in the emergency area. Therefore, IEEE 802.11af is better suited for our architecture.

\section{High-Tier Access Network}

The third tier is formed by the existing radio networks used for surveillance or monitoring such as TETRA and TETRAPOL or commercial GSM/LTE network, satellite network, or even Wi-Fi hot spots as shown in Figure 1. For backhaul access, ICGs forward data through other ICGs until a contact with one of these backhaul networks can be established and then exploit these existing infrastructure.

The deployment of ICGs mainly includes the establishment of static ICG sites to provide the coverage in all the possible regions where the PPDR organizations potentially operate.

According to the capacity requirements, the deployment also ensures that the ICG network has sufficient connectivity with the backhaul networks connecting to PPDR remote stations. Moreover, the self-configuration property of the communication protocols developed for ICG network allow the mobile ICGs to join the network spontaneously to overcome the possible failures of existing nodes or extending the coverage area within no time. Thus, the ICG network aims to enhance the existing infrastructure of public (TETRA), commercial (LTE), and satellite networks. Moreover, ICGs also facilitate PPDR applications by supporting multiple fixed spectrum interfaces in addition to OSA for ubiquitous communication. Furthermore, the ability to self-configure and adaptively change communication parameters provides robustness and sustainability, which is much needed in disaster relief scenarios.

\section{PPDR Communication Framework}

Figure 2 demonstrates the system level approach for designing communication protocols at different tiers of the proposed PPDR architecture. Data from different applications running on PPDR network is classified using traffic classifier layer according to the priority of the application or a particular scenario to achieve minimum time of delivery of event from the network layer, where event has been detected to decision point. Early warning system is based on micro-fusion of data supplied by local sensing element and traffic classifier output. A QoS framework based on the communication requirements 
of PPDR data maps the classified traffic to different traffic queues for transmission and ensures that the required QoS is provided to each traffic class.

Network layer and other lower layers of ISO/OSI provide addressing mechanism, error correction, and link layer functionality, and are responsible to route data to a base-station using appropriate ICGs. SDN can be used here. However, SDN has centralized approach [18], whereas ICG network has adhoc structure. Moreover, such centralized approaches have single point of failure problem. Therefore, SDN is more suitable for a higher layer, and may especially prove valuable for interoperability, by controlling and coordinating the flows of different first responder units.

Middle tier forming PPDR infrastructure is built by the deployment of a number of ICGs in pre-disaster arrangement as well as the on-scene deployment of ICGs. These ICGs implement self-configuration protocol to enable flexible and resilient PPDR architecture. They also implement ad hoc routing protocol to dynamically determine the route according to the availability of the infrastructure. Essentially they are relay nodes, which forward application data from lower tier to application base-station. Since the proposed architecture is an enhancement to the existing PPDR infrastructure, it integrates existing commercial and public wireless network into our architecture by adding an interface to ICG. Therefore, it enables to exploit the pre-installed communication infrastructure using UDP or TCP/IP protocol for accessing the application base-station. While communication between the ICGs is performed over cognitive radio interface, this makes the ICG middle tier network spectrum efficient and resilient. Its functions are divided into the following layers:

- Relay layer acts as a relay and builds the data to an IP packet for transmission to higher tier.
- QoS framework implements the services that include mapping the lower tier QoS functions to higher tier QoS functions, prioritizing interfaces, etc.

- Intelligent Network layer performs the task of finding route and appropriate interface to send data to base station. This may involve coordination with other ICGs.

- Interface manager maintains and monitors the status of each interface and acts as a single access point to higher layer. It also shields all the underlying interfaces' complexity to simplify the design of higher layers.

\section{Comparison of Architectures}

In this section, we aim to present a comparison of the proposed architecture with other PPDR architectures in the literature. We investigate six criteria that we believe is important for future PPDR networks.

1) Interoperability indicates that the architecture supports multiple network interfaces of various first responder units.

2) Expansion is the ability to increase coverage on demand.

3) Anti-jamming is required for terrorist attacks.

4) Recovery is the capability to cover for failed units in the architecture.

5) Deployment indicates whether the architecture is infrastructure-based or ad-hoc.

6) Broadband capability is the finally criterion we are interested in.

We present comparison of our architecture with four other architectures, the TETRA Enhanced Data Services (TEDS), LTE-based solution proposed in [1], Satellite-based architecture in [3] and cognitive radio capable solution in [4].
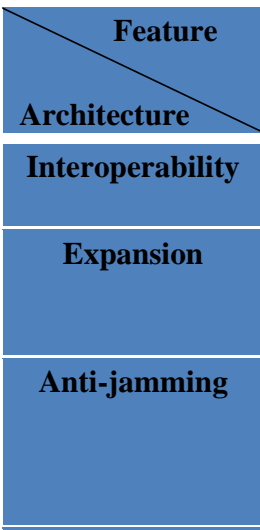

Recovery

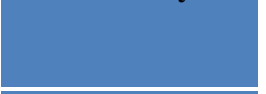

Deployment

Broadband

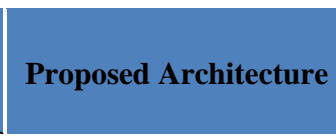

Yes (multi-interface)

Yes (due to mobile ad hoc ICG nodes)

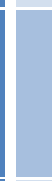

Cognitive spectrum provides more resilience against jamming or attack Easy recovery in disaster (multi-hop and mesh connectivity)

Infrastructure/Adhoc

$$
\text { Yes }
$$
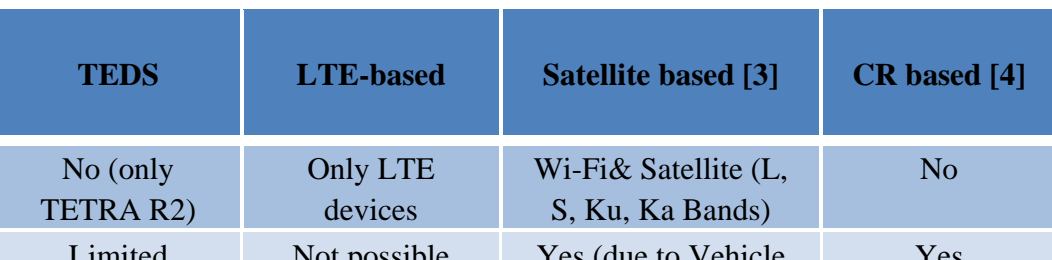

Only LTE
devices

Wi-Fi\& Satellite (L,

$\mathrm{S}, \mathrm{Ku}, \mathrm{Ka}$ Bands)

Not possible

Yes (due to Vehicle

Coomunication

Gateway nodes)

Fixed spectrum,
the jammed
bands cannot be
restored
Might be

Fixed spectrum,

the jammed

bands cannot be restored

\section{Might be} unavailable in unavailable in disaster

\begin{tabular}{|c|} 
Infrastructure \\
Wideband (upto \\
450kbps)
\end{tabular}

disaster

Infrastructure

\section{Fixed spectrum, the jammed bands cannot be restored \\ Limited (no multi- hop)}

Yes
Infrastructure/Adhoc

No

Yes

Table 2 Comparison of existing work with our architecture 
As summarized by Table 2, our solution covers the widest range of features. The new TETRA network architecture has problems mainly due to its strict infrastructure based architecture. It lacks important capabilities such as data forwarding and dynamic spectrum access. Furthermore, despite the improvements, it still has insufficient bandwidth for future PPDR networks which have high bandwidth demands (e.g., for real-time video). LTE-based approach suffers from similar inadequacies except for bandwidth. The architecture proposed in [3] offers some flexibility by use of mobile access points. However, it cannot offer anti-jamming since it does not have CR capability, and only offers limited recovery since it does not support multi-hop communication. The CR-based solution does not support data links and like the others, does not have interoperability support.

To highlight the capabilities of ICG, we analyze a hypothetical case. ICGs are deployed in a building, before disaster. We present a simple analysis of the communication area inside the rubble that can be covered by ICGs. We take the path loss as $7 \mathrm{~dB}$ and $4 \mathrm{~dB}$, for concrete and brick, respectively, for a thickness of $0.1 \mathrm{~m}$.

We have evaluated path loss of these materials and found it to be linear in the log scale. These results are in line with those in the literature (e.g. [19]). We assume a receiver sensitivity of $-110 \mathrm{dBm}$, transmitter and receiver antenna gains of $30 \mathrm{dBi}$. Transmission power of $50 \mathrm{dBm}$ is assumed to be used in emergency situation. This allows for a total attenuation of 190 $\mathrm{dB}$. We realize that this power value is much higher than allowed 2W EIRP in Europe and 4W EIRP in US. However, ICGs will only use this high transmission power mode for emergency rescue operations, for a very short time to collect data from the event area. Therefore, we think this is a reasonable assumption.

In Figure 3, we show the amount of power needed to transmit successfully to a $5 \mathrm{~m}$ distance for various percentages of concrete and brick in the rubble. For a high amount of cases, ICGs can transmit data when they are $5 \mathrm{~m}$ deep in the rubble.

\section{Simulation Results}

In this section, we provide the results of the simulations we performed to demonstrate the effectiveness of our architecture. We assume a disaster scenario where a certain emergency situation (fire, bombing, etc.) occurs in the middle of the
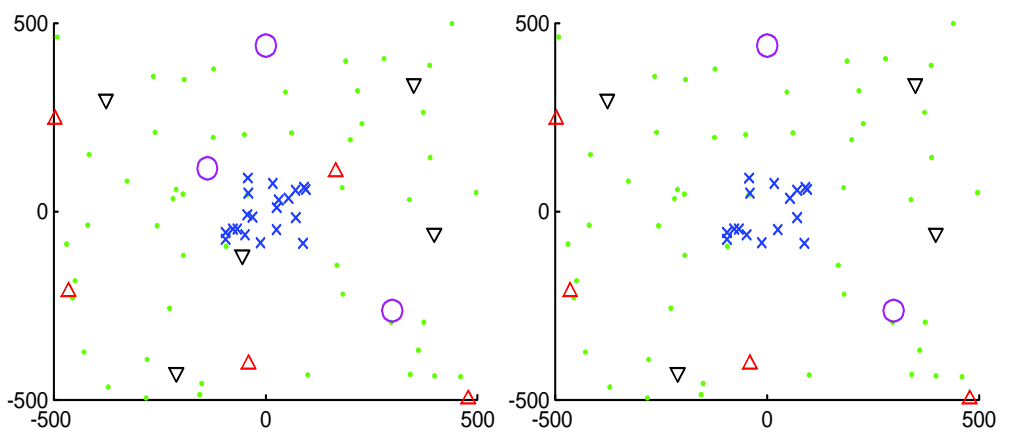

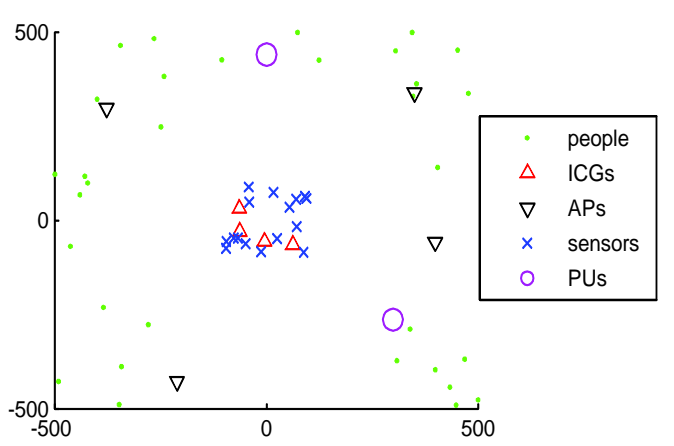

Figure 3 Power required to transmit to $5 \mathrm{~m}$ for various material percentages

simulation area, which is taken to be $500 \mathrm{~m} \times 500 \mathrm{~m}$. People, sensors, ICGs, and primary users (PU) are located randomly according to a Poisson Point Process within the area. Since we are only interested in the sensors located in the event area, we focus on sensors within $100 \mathrm{~m}$ of the event.

To compare the effectiveness of ICG, we also place equal number of non-mobile Wi-Fi access points (APs) inside the area. We beleive five APs inside a $500 \times 500 \mathrm{~m}$ area is a reasonable assumption. We choose $\mathrm{Wi}-\mathrm{Fi}$ for comparison, because centralized systems such as cellular has the problem of single-point-of-failure, which is critical for disaster scenarios. We assume a channel bandwidth of $20 \mathrm{MHz}$ for APs, as suppored by most IEEE 802.11 variants. Assuming ICGs use TV whitespaces, we take $6 \mathrm{MHz}$ as channel bandwidth for ICGs. Since nowadays most TV users are cable subscribers, The number of TV users that use over-the-air antennas. Therefore, we assume three PUs.

After the event, some of the devices are destroyed with probabilities inversely proportional to their distance to the event. Furthermore, people and ICGs start to move. We assume the "gravitational" mobility model proposed in [20], i.e. people move away from the event area with velocities inversely proportional to their distance to the event, and ICGs move towards the event area with velocities proportional to the square of the distance to the event.

Figure 4 Sample positions for simulation elements 


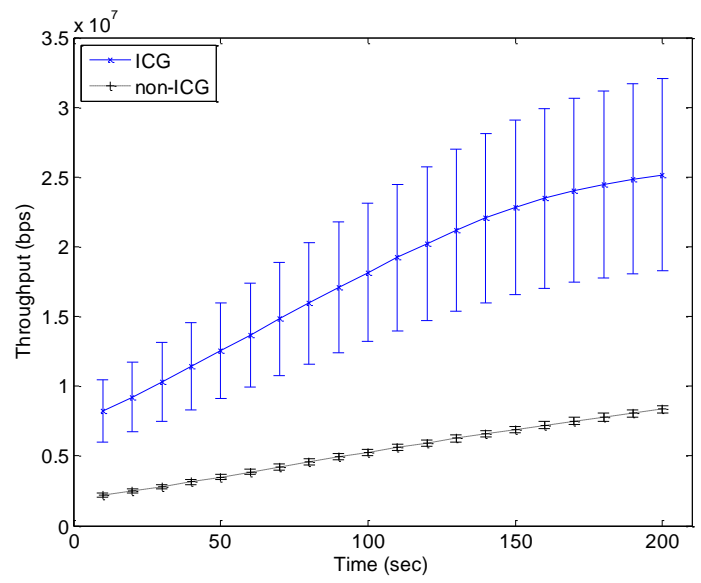

Figure 5 Throughput vs time

We compare the throughput of data gathered from the sensors by ICGs and APs, by repeating the same scenario for both cases. We run the simulation 1000 times with different random placements. For each placement, we assume PU existence probabilities (probability of a PU being active), from 0.1 to 0.9 .

In Figure 4, we show sample random location distributions before the event, right after the event and at simulation end, i.e., 200 seconds after the event, respectively. By comparing these figures, we see that some of the equipment gets destroyed in the event. Also, people have moved away from the event area and ICGs have moved closer by the end of 200 seconds.

We present the throughput obtained by both ICGs and APs as time passes in Figure 5. ICGs provide higher throughput even though their channel bandwidth is considerably lower (6 $\mathrm{MHz}$ vs. $20 \mathrm{MHz}$ ). As time progresses, people move away from the area, reducing both the number of users served by APs and the interference on APs. Therefore, AP throughput increases with time. However, ICG throughput increases more rapidly, since ICGs can get closer to the sensors and further increase their SINR. The wider deviation on ICG throughput is due to different PU existence probabilities.

The effect of PU existence probability is presented in Figure 6. We see that ICGs perform better up to PU existence probability of 0.85 . This shows that our proposed architecture enables higher throughput for majority of the cases due to its cognitive radio and mobility capabilities.

\section{Conclusions}

In this paper, we introduce an adaptable and resilient architecture that enables sustainable communication and timely gathering of crucial event data and its delivery to the responder units at the site as well as the command and control centers that are off-site. We lay out communication requirements and challenges that should be addressed by the next-generation PPDR network architecture and explained how the proposed solution fulfills these requirements.

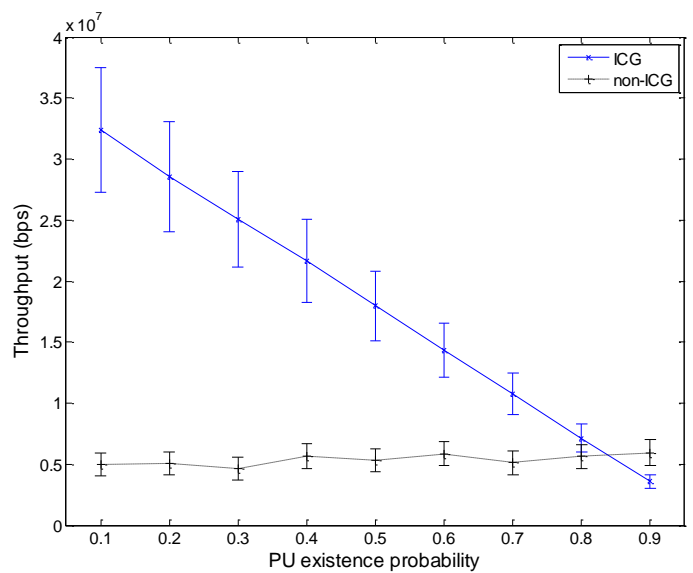

Figure 6 Throughput vs PU existence probability

\section{References}

[1] R. Ferrus, O. Sallent, G. Baldini, L. Goratti, "LTE: the technology driver for future public safety communications," IEEE Communications Magazine, vol.51, no.10, pp.154,161, October 2013.

[2] J. Rajamaki, "Redundant Multichannel Public Safety Communication Network for Public Protection and Disaster Relief (PPDR) Organizations", In Proc. Mathematical Modelling and Simulation in Applied Sciences, pp. 56-61, 2012.

[3] G. Iapichino, C. Bonnet, O. Herrero, C. Baudoin, I. Buret, "A mobile ad hoc satellite and wireless Mesh networking approach for Public Safety communications," in Proc. SPSC'08, pp.1-6, 2008.

[4] W. Wang, W. Gao, X. Bai, T. Peng, G. Chuai, W. Wang, "A Framework of Wireless Emergency Communications based on Relaying and Cognitive Radio," In Proc. of IEEE 18th International Symposium on Personal, Indoor and Mobile Radio Communications (PIMRC 2007), pp. 1-5, Sept. 2007.

[5] M. T. Gardner, C. Beard, D. Medhi, "Using network measure to reduce state space enumeration in resilient networks," Design of Reliable Communication Networks (DRCN), 2013 9th International Conference on the , vol., no., pp.250,257, 4-7 March 2013.

[6] European Network and Information Security Agency (ENISA), "Measurement Frameworks and Metrics for Resilient Networks and Services: Technical Report," https://www.enisa.europa.eu/activities/Resilience-and-

CIIP/Incidents-reporting/metrics/reports/metrics-tech-report last accessed Feb. 2015.

[7] T. Winderl, "Disaster Resilience Measurements", UNDP Report, Feb 2014. http://www.ieee802.org/3/ah/index.html last accessed Feb 2015.

[8] L. Sadeghioon, P. Gravey, A. Gravey, "Resilience in transparent OPS multi-rings," Photonics in Switching (PS), 2012 International Conference on , vol., no., pp.1,3, 11-14 Sept. 2012.

[9] P. Smith, A. Schaeffer-Filho, D. Hutchison, A. Mauthe, "Management patterns: SDN-enabled network resilience management," Network Operations and Management Symposium (NOMS), 2014 IEEE , vol., no., pp.1,9, 5-9 May 2014.

[10] J-P. Javaudin, M. Bellec, "OMEGA project: On Convergent Digital Home Networks," Cross Layer Design (IWCLD), 2011 Third International Workshop on, vol., no., pp.1,5, Nov. 30 2011-Dec. 12011.

[11] Y-J. Luo, X. Yang, X. Zhang, "An Effective Resilient Data Aggregation Algorithm in Wireless Sensor Networks," Wireless Communications, Networking and Mobile Computing, 2007. WiCom 2007. International Conference on , vol., no., pp.2642,2645, 21-25 Sept. 2007 
[12] E. Onem, S. Eryigit, T. Tugcu, A. Akurgal, "QoS-Enabled Spectrum-Aware Routing for Disaster Relief and Tactical Operations over Cognitive Radio Ad Hoc Networks," Military Communications Conference, MILCOM 2013 - 2013 IEEE , vol., no., pp.1109,1115, 18-20 Nov. 2013.

[13] X. Li, D. Qiao, Y. Li, H. Dai, "A novel through-wall respiration detection algorithm using UWB radar," Engineering in Medicine and Biology Society (EMBC), 2013 35th Annual International Conference of the IEEE, vol., no., pp.1013,1016, 3-7 July 2013.

[14] M. Chiani, A. Giorgetti, "Coexistence Between UWB and Narrow-Band Wireless Communication Systems," Proceedings of the IEEE, vol.97, no.2, pp.231,254, Feb. 2009

[15] E. T. Northardt, I. Bilik, Y. I. Abramovich, "Spatial Compressive Sensing for Direction-of-Arrival Estimation With Bias Mitigation Via Expected Likelihood," Signal Processing, IEEE Transactions on , vol.61, no.5, pp.1183,1195, March1, 2013.

[16] O. B. Akan, O. B. Karli, O. Ergul, "Cognitive Radio Sensor Networks," IEEE Network, vol. 23, no.4, pp. 34-40, July 2009.

[17] M. H. Rehmani, A. C. Viana, H. Khalife, S. Fdida, "A cognitive radio based Internet access framework for disaster response network deployment," Applied Sciences in Biomedical and Communication Technologies (ISABEL), 2010 3rd International Symposium on , vol., no., pp.1,5, 7-10 Nov. 2010.

[18] P. Fonseca, R. Bennesby, E. Mota, A. Passito, "A replication component for resilient OpenFlow-based networking," Network Operations and Management Symposium (NOMS), 2012 IEEE , vol., no., pp.933,939, 16-20 April 2012.

[19] D. Pena, R. Feick, H. D. Hristov, W. Grote, "Measurement and modeling of propagation losses in brick and concrete walls for the 900-MHz band," Antennas and Propagation, IEEE Transactions on , vol.51, no.1, pp.31,39, Jan 2003.

[20] S. C. Nelson, A. F. Harris, R. Kravets, "Event-driven, role-based mobility in disaster recovery networks," ACM workshop on Challenged networks, pp. 27-34, 2007.

Ozgur Ergul [S'11] received his M.S. degree in electrical and electronics engineering from Middle East Technical University, Turkey, in 2006. He is currently pursuing his Ph.D. degree as a research assistant in the Next-generation and Wireless Communications Laboratory, Koc University. His current research interests include cognitive radio networks, wireless sensor networks and Internet of Things.

Ghalib A. Shah [M'09] received his PhD degree in computer engineering from Middle East Technical University, Turkey in 2007. He is currently a visiting foreign professor at AlKhawarizmi Institute of Computer Science, UET Lahore. His research interests include the design and analysis of communication protocols from MAC to Transport layer for cognitive radio networks, wireless multimedia networks, Internet of Things and software defined networks.

Berk Canberk [M'13] received his PhD degree in Computer Engineering from Istanbul Technical University, Turkey, and his MSc degree in Digital Communications Engineering from Chalmers University of Technology, Sweden, in 2011 and 2005 respectively. He was a post-doc scholar in Broadband Wireless Networking Laboratory (BWNLab) at the School of Electrical and Computer Engineering, Georgia Institute of Technology, Atlanta, USA, in 2011. He was also a research scholar in BWNLab during 2008-2009. He is currently an assistant professor in the Computer Engineering Department of Istanbul Technical University. He is an editor in IEEE Transactions in Vehicular Technology, area editor in ELSEVIER Computer Networks and associate editor in WILEY International Journal on Communication Systems. Dr. Canberk is the recipient of "Best Poster Paper Award in IEEE INFOCOM 2015" and "Turkish Telecom 2013 Colloborative Research Award". He has been involved in several international conferences as technical program co-chair, regional chair, publicity chair, tutorial chair and TPC member. His current research interests include software defined wireless networking, energy-aware cognitive radio network management and next generation LTE networks.

Ozgur B. Akan [M'00, SM'07] received his Ph.D. degree in electrical and computer engineering from the Broadband and Wireless Networking Laboratory, School of Electrical and Computer Engineering, Georgia Institute of Technology in 2004. He is currently a full professor with the Department of Electrical and Electronics Engineering, Koc University and the director of the Next-generation and Wireless Communications Laboratory. His current research interests are in wireless communications, nano-scale and molecular communications, and information theory. 